\title{
Corrugated long period fiber gratings as band-rejection filters
}

\author{
C. Y. Lin and L. A. Wang \\ Institute of Electro-Optical Engineering \\ National Taiwan University \\ Taipei, Taiwan, R.O.C. \\ Address of Correspondent \\ Lon A. Wang \\ Department of Electrical Engineering, Rm. 539 \\ National Taiwan University \\ Taipei, Taiwan, R.O.C. \\ TEL:(886-2)23635251 \\ FAX:(886-2)23656327 \\ Email: lon@ccms.ntu.edu.tw
}

Applications such as gain-flattening in an erbium-doped fiber amplifer (EDFA) require wavelength dependent loss element with very low back reflection. Long period fiber gratings (LPFG's) which operate in the transmission mode have found a niche in these applications. However the losses in the conventional LPFG's are almost fixed so that gain-flattening can only work at specific conditions [1]. Here we propose a new LPFG made of corrugated structure to form a loss tunable band-rejection filter. The devices may provide spectral shaping flexibility for dynamically flattening gain spectrum of an EDFA.

A dispersion shifted fiber with an original cladding diameter $125 \mu \mathrm{m}$ is used. Thin layers of metals are coated onto the fiber to form a segmented ring pattern for etching protection when further immersed in hydrofluoric acid solution. The uncoated cladding sections are etched to a reduced diameter as shown in Fig. 1. The corrugated structure has a period of $400 \mu \mathrm{m}$ over a total length of $20 \mathrm{~mm}$. When the etched section is placed straight, an effective waveguide index modulation results due to the corrugated structure. Such a modulation is very small unless the sections are etched close to the core. The coupling efficiency between core and cladding modes is therefore very small. However, when a stress is applied, a much stronger index variation is induced in the corrugated structure This is because the strain is inversely proportional to the square of diameter [2], and the induced index variation resulted from photoelastic effect is related to the difference of core and cladding diameters. The index variation increases with stress and results in a LPFG with increasing resonant loss, thus allowing loss tunability. The transmission 


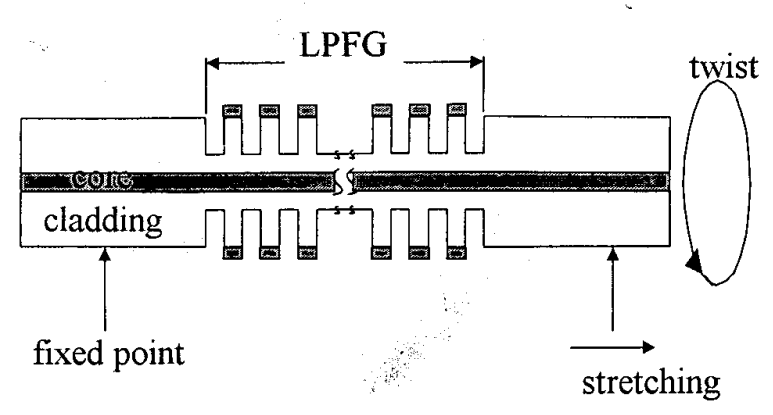

Fig. 1: Schematic diagram of a corrugated LPFG under stress and twist

spectra of LPFG with different stresses are shown in Fig. 2. It is seen that the transmission loss at the resonance peak increases with stress because the index difference in regions of etched and unetched becomes larger. Note the resonant wavelength $t$ is almost unshifted during the overall loss tuning, and the maximum loss can be tuned to as high as $25 \mathrm{~dB}$.

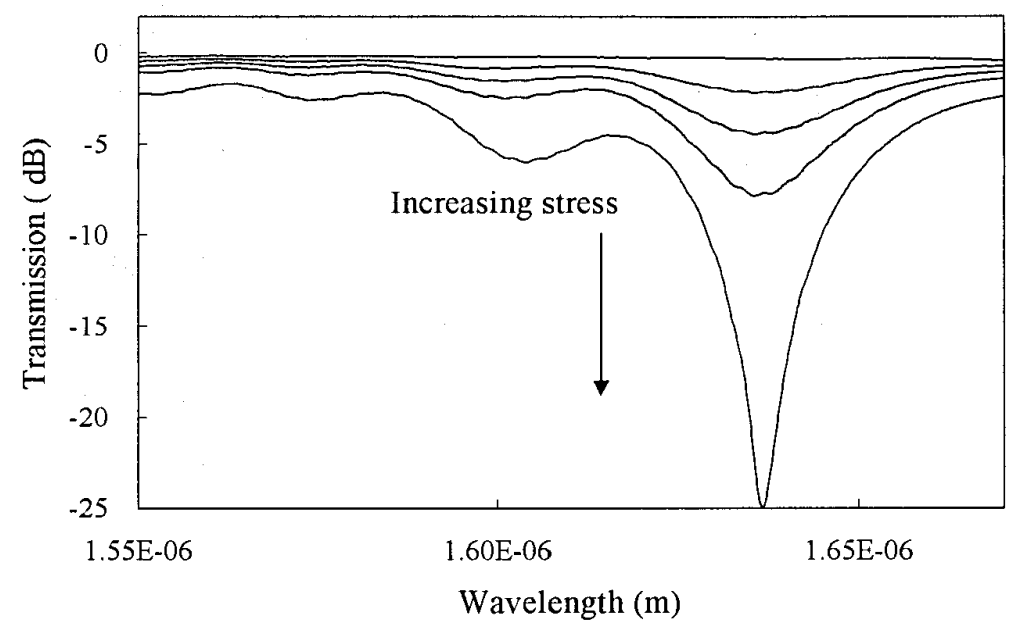

Fig.2: Evolution of transmission spectra when strains are applied to the LPFG

For some applications, a band-rejection filter operating over a wide wavelength range is desired which requires the filter have wavelength tunability. Fig. 3 shows the resonant peak wavelength can be tuned by twisting the LPFG. This wavelength tunability when combined with the loss tunability mentioned previously may serve for flattening the gain of an EDFA. 


\section{$22 / \mathrm{TuB} 1-3$}

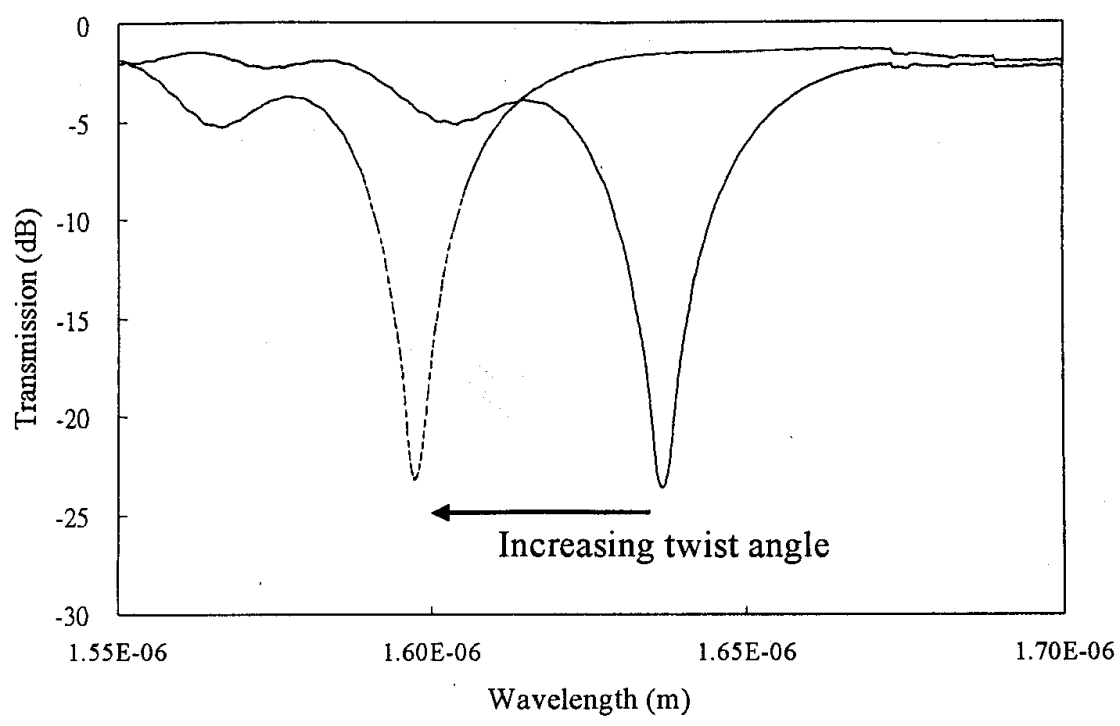

Fig.3: Evolution of transmission spectra when twists are applied to the LPFG

In summary, we demonstrate, for the first time to our knowledge, a new technique to fabricate a loss and wavelength tunable LPFG by varying the stress and twist applied to a corrugated structure where the LPFG is defined. Such a tunable band-rejection filter may find useful applications in optical fiber communication such as gain-flattening of an EDFA.

1. Vengsarkar, A.M. , Pedrazzani, J.R., Judkins, J.B., and Lemaire, P.J. Optics. Lett., 1996, 21, pp. 336-338

2. Song, M., Lee, B., Lee, S. B. and Choi, S. S. Optics Lett., 1997, 22, pp. 790-792 